\title{
Experimental Rat Model of Bony Defects in the Facet Joint Maintained with Bone Wax for the Study of Spinal Pain
}

\author{
Jinyoung Oh $\mathbb{D}^{1, *}$, Daehyun Jo $\mathbb{D}^{2, *}$, Kicheol Park $\mathbb{D}^{3}$, Posoon Kang ${ }^{4}$, Youngsup Shin $\mathbb{D}^{5}$ \\ 'Department of Anesthesiology and Pain Medicine, Kyungpook National University Chilgok Hospital, School of Medicine, Kyungpook National \\ University, Daegu, Republic of Korea; ${ }^{2}$ Jodaehyun Pain Center, Jeonju, Republic of Korea; ${ }^{3}$ Clinical Research Institute, Daejeon St. Mary's Hospital, The \\ Catholic University of Korea, Daejeon, Republic of Korea; ${ }^{4}$ Department of Anesthesiology and Pain Medicine, Konynang University Hospital, Daejeon, \\ Republic of Korea; ${ }^{5}$ Department of Anesthesiology and Pain Medicine, Chungnam National University College of Medicine, Daejeon, Republic of \\ Korea
}

Correspondence: Youngsup Shin, Department of Anesthesiology and Pain Medicine, Chungnam National University College of Medicine, 282, Munhwa-ro, Junggu, Daejon, 350I5, Republic of Korea, Tel +82-42-280-7840, Fax +82-42-280-7968, Email ysshin@cnu.ac.kr

*These authors contributed equally to this work

Purpose: Studies using experimental rat models for low back pain due to facet-joint defects are scarce. This study used a novel experimental rat model to determine whether bony defects induced by facetectomy could be maintained by bone wax, thus mimicking spondylolysis, and to analyze the effect of the facetectomy on rat behavior.

Patients and Methods: Twelve 10-week-old male Wistar rats weighing 300-350 g were divided into group A $(n=6)$ that underwent unilateral facetectomy of the right L5-6 facet joint and group B $(n=6)$ that additionally applied water-soluble bone wax at the facetectomy site. The difference in the left and right stride length, detected by the footprint test, and change in the left and right facet joint area were compared before and 4 weeks after the experiment.

Results: Even though the difference between the left and right stride lengths of groups A and B was not statistically significant, in contrast to group A, group B showed a shorter stride length on the right side ( $p=0.22$ and 0.46 , in group A and group B, respectively). The right facet joint area, where the facetectomy was performed, was significantly smaller in group B 4 weeks after surgery, but not in group A ( $\mathrm{p}=0.50$ and $<0.01$, in group A and group B, respectively).

Conclusion: Based on the results, we concluded that the bony defects, induced by facetectomy at the L5-6 facet joint, were maintained with bone wax. This study will provide an experimental model for bony defects in the facet joint.

Keywords: facet-joint defect, bone wax, Masson's trichrome stain, footprint test, spondylolysis, rat

\section{Introduction}

Facet joints are a major source of spinal pain. It is estimated that in more than $30 \%$ of patients with lumbar spinal pain, the pain originates from the facet joint. ${ }^{1}$ Most cases of low back pain originating from the facet joint are related to its degeneration. ${ }^{2}$ Some cases are related to a bony defect in the facet joint called spondylolysis. ${ }^{3}$

Spondylolysis is defined as a defect or fracture of the pars interarticularis. It is often caused by mechanical stress and is common in children and adolescents with low back pain. ${ }^{3}$ It is not clinically significant during early stages, but repetitive forces cause minute damage to the bone over time; when this rate of damage is more rapid than the cellular repair of the bone, a fracture of the facet joint occurs. This condition is observed in $25-39 \%$, and even as high as $47 \%$, of athletes with back pain. ${ }^{3,4}$ Pain due to spondylolysis generally has an insidious onset and recurrence and is associated with activity. It generally worsens with back extension and occasionally radiates to the buttock or posterior thigh, which makes it similar to the facet joint syndrome. ${ }^{5-7}$ 
Most patients with spondylolysis have a favorable prognosis with conservative treatment, such as wearing a brace and refraining from sport activities during the early stage. ${ }^{8,9}$ However, bony defects in spondylolysis can be followed by spinal instability. When instability from spondylolysis results in the translation of the vertebral body, displacement of the vertebra can occur, which is called spondylolisthesis. Several studies showed that approximately $15 \%$ of the cases of spondylolysis progress to spondylolisthesis. ${ }^{10-12}$

Currently, surgery is the only way to repair bony defects. Fixation of the bony defect site using screws, with or without rods, has promising results. ${ }^{6}$ However, various non-invasive or minimally invasive modalities are emerging, especially for the spine. Interventions such as percutaneous injection techniques can relieve back pain in most patients. Low-intensity pulsed ultrasound can be considered for spondylolysis; however, further studies are necessary to determine its value. ${ }^{6}$ Several interventions for bony defects involving regenerative materials and percutaneous techniques have been developed, and they have the potential to be applied to this condition. However, further in vivo studies are necessary to adopt these new treatments in clinical practice.

Animal models are useful for providing basic evidence of new therapeutic concepts and spinal diseases. Various biological treatment modalities have been suggested for intervertebral disc degeneration based on animal models of induced degeneration of the intervertebral disc. ${ }^{13}$ Stem cell therapy for neuropathic pain has also been proposed based on animal studies. ${ }^{14}$

The animal models used to study the pathophysiology and treatment of spondylolysis in previous studies were mainly large; they included pigs, calves, and sheep. ${ }^{15-17}$ The advantage of using them is that their conditions are similar to those of humans; however, they are not easy to obtain and have limitations in various experiments. In contrast, rat models are easier to purchase and manage; therefore, various experiments can be performed on a larger number of animals.

Several rat studies using lumbar facetectomy have been reported. Sakamaki et al proposed a rat model in which spinal destabilization was induced through L5 laminectomy and bilateral L5-6 facetectomy (posterior destabilization surgery). ${ }^{18}$ However, they did not evaluate the structural defect but studied the instability of the spine following posterior destabilization surgery; essentially, their model was for spondylolytic spondylolisthesis, which is a progressive form of spondylolysis. Afterwards, Fukui et al reported other experiments in which lumbar facetectomy was performed in rats. They investigated the behavioral changes (ie, gait disturbance) and degenerative changes of the intervertebral disc and changes of spinal alignment after bilateral L4-5 facetectomy. ${ }^{19-21}$ However, they also did not investigate the facet joint defect itself.

In the previous studies, a bony defect was induced through facetectomy, but the defect itself was not evaluated. The bone is a dynamic tissue that undergoes remodeling, and an artificially created defect recovers spontaneously if the size is not critical. ${ }^{22-24}$ Bone morphological analyses using microCT in the study by Fukui et al showed no significant difference between the Experimental (only exposure of the paravertebral muscles of bilateral facet joints at the L4-5 level) and Sham (complete resection of bilateral L4-5 facet joints) groups in blood volume fraction. ${ }^{20}$ For the interventional studies on the treatment of bony defects in the spine, a model that resembles the disease state should be established.

In this regard, we devised use of bone wax to maintain the artificial bony defect. Bone wax is a hemostatic agent widely used in surgeries. It coats the bleeding bone surfaces and provides a mechanical tamponade to prevent blood from oozing out. ${ }^{25}$ The interruption of bone healing is a major complication of bone wax; however, this limitation can be utilized to maintain bony defects. ${ }^{26}$

We conducted this study to determine whether bony defects induced by facetectomy could be maintained by bone wax using a rat experimental model of bony defects in facet joints and to analyze the effect of the defect on rat behavior. Our results indicate that applying bone wax at the site of the facetectomy can maintain the bony defect created by the facetectomy, which is similar to the defect in the pars interarticularis during spondylolysis.

\section{Materials and Methods}

\section{Animals}

Twelve 10-week-old male Wistar rats weighing 300-350 g were obtained from Samtako BioKorea Co. Ltd (Osan-si, Gyeonggi-do, Republic of Korea). They were housed in individual cages in a room with controlled temperature ( $23 \pm$ $\left.2{ }^{\circ} \mathrm{C}\right)$ and humidity $(55 \% \pm 10 \%)$ and a light-dark cycle of $12: 12 \mathrm{~h}$. The animals had free access to food and water. The 


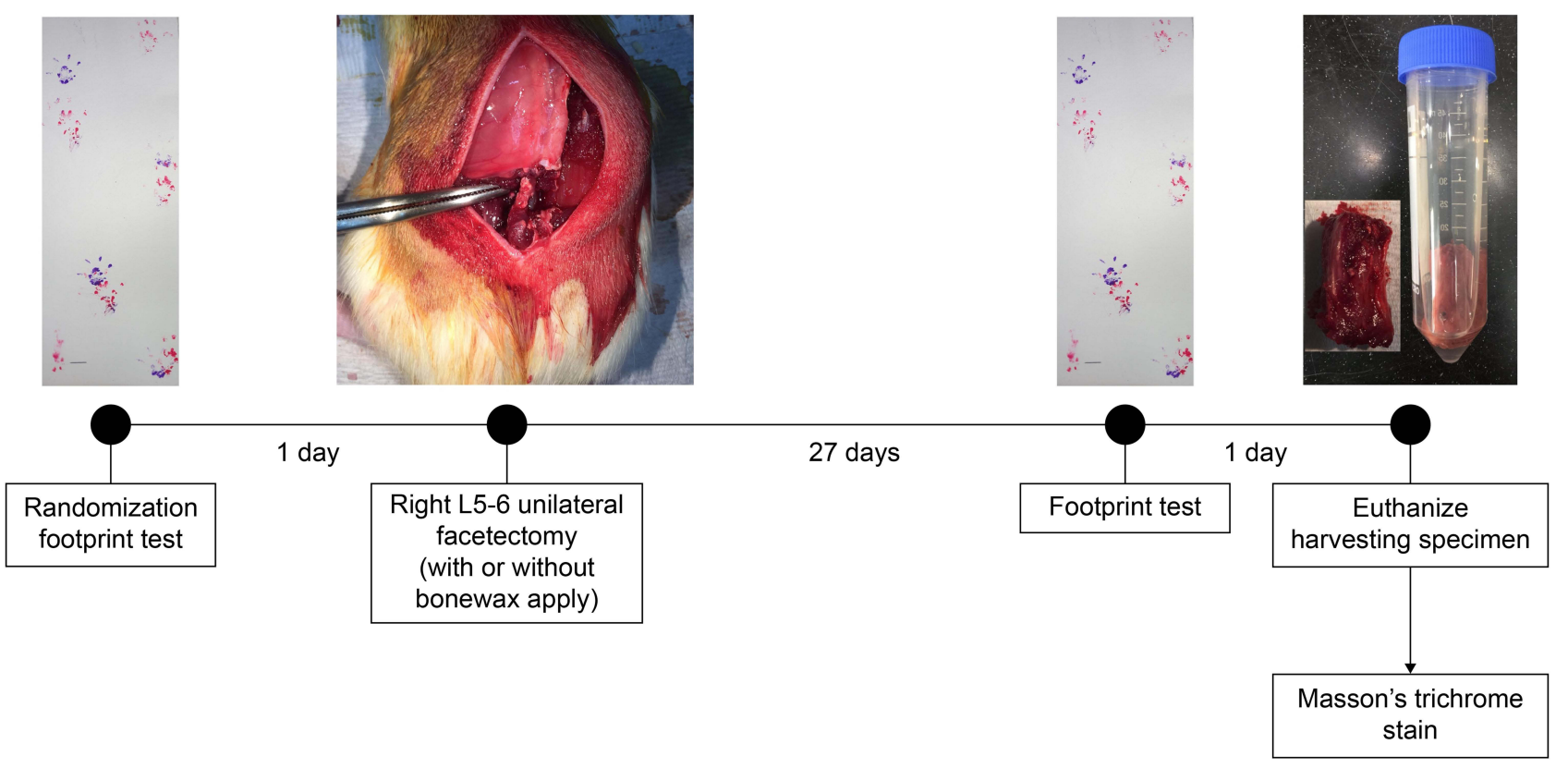

Figure I The process (time course) of the experiment.

protocol for this study conformed to the guidelines of our institution for the care and use of laboratory animals. All study procedures were approved by the Institutional Review Board of Daejeon St. Mary's Hospital, The Catholic University of Korea (CMCDJ-AP-2016-007). The study process is summarized in Figure 1.

\section{Surgical Procedure: Facetectomy}

We randomly divided the rats into groups A (the control group) and B (the bone wax group); each group contained six rats. All the rats underwent unilateral facetectomy at the right L5-6 level. No treatment was done after the facetectomy in group A, whereas water-soluble bone wax (Ostene, Ceremed, Inc., Los Angeles, CA) was applied to the facetectomy site in group B. The rats were anesthetized using a $30 \mathrm{mg} / \mathrm{kg}$ intraperitoneal injection of a combination of tiletamine and zolazepam (Zoletil 100, Virbac Sante Animale, Carros, Provence-Alpes-Côte d'Azur, France) and $10 \mathrm{mg} / \mathrm{kg}$ xylazine (Rumpune 10mL, Elanco, Ansan-si, Gyeonggi-do, Republic of Korea).

A midline skin incision was made on the back of each rat extending from L1-L6. The vertebral levels were identified by counting from L1, which is just below T12 where the ribs are attached. The paravertebral muscles were detached from the L5 and L6 laminae to expose the L5-6 facet joint. A right L5-L6 facetectomy was performed using a bone cutter. Thereafter, bone wax (Ostene) was applied to the facetectomy sites of the rats in group B; Ostene comprises watersoluble alkylene oxide copolymers and is a water-soluble implant material approved by the Food and Drug Administration to control bleeding from bone surfaces (Figure 2). The paravertebral muscles, subcutaneous tissue, and skin were sutured layer-to-layer using 4-0 silk. To prevent infection and pain control, $20 \mathrm{mg} / \mathrm{kg}$ of gentamycin (Samu gentamycin injection 50mL vial, Samu Mediain Co., Ltd., Yesan-Gun, Chungcheongnam-do, Republic of Korea) and $5 \mathrm{mg} / \mathrm{kg}$ of ketorolac (UNI-Ketopro, Ubtech ${ }^{\circledR}$, Anyang-Si, Gyeonggi-do, Republic of Korea) were injected subcutaneously before the surgery and once daily for 3 days after the surgery. During this period, the surgical wounds were dressed using povidone and alcohol. The rats were housed in the same environment as before the surgery, and they were euthanized with $\mathrm{CO}_{2}$ four weeks later. ${ }^{27}$

\section{Masson's Trichrome Stain}

The lumbar spines at the L5-6 level were harvested after euthanasia 4 weeks after surgery. After a midline skin incision and detachment of the paravertebral muscles, the exposed vertebrae were separated from the soft tissues. We identified the L5 and L6 vertebrae and separated them from the vertebral column using a rongeur. 

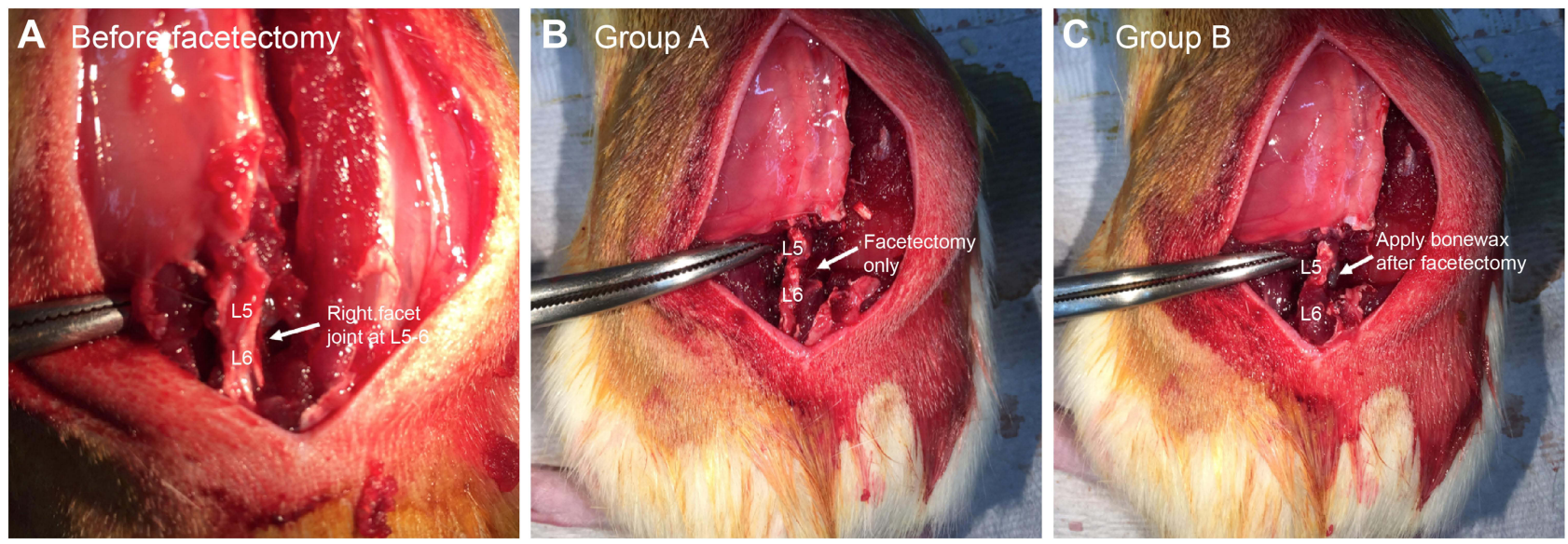

Figure 2 Facetectomy of the right L5-6 facet joint. (A) Normal facet on right L5-6. (B) In group A, subcutaneous tissue and skin were closed immediately after right facetectomy, and the surgery was completed. (C) In group B, bone wax was applied to the facetectomy site, the subcutaneous tissue and skin were closed, and the surgery was completed. The images are those of Sample \#2 from Group A and Sample \#I from Group B.

The specimens were fixed with $4 \%$ paraformaldehyde $(\mathrm{pH} 7.2$, room temperature) for $48 \mathrm{~h}$ and decalcified using a decalcifying solution with ethylenediaminetetraacetic acid (Sigma-Aldrich, Missouri, USA) for 2 weeks, followed by embedding in paraffin. The tissues were cross-sectioned transversely (in axial plane) into 4- $\mu \mathrm{m}$ thick sections using a rotation microtome (Thermo Fisher Scientific, Massachusetts, USA) and stained with Masson's trichrome stain (Masson's trichrome stain kit, Polysciences, Inc., Warrington, USA) according to the manufacturer's protocol to evaluate the mineralized bone matrix and osteoid. ${ }^{28}$

\section{Quantification and Analysis}

After the staining was completed, we photographed a slide using a microscope (Eclipse TE300 inverted microscope, Nikon ${ }^{\circledR}$, Minato, Tokyo, Japan) and its software. For each specimen, one slide with the best observation of the left facet joint (the slide with the largest area of the left facet joint), which did not undergo facetectomy, was selected for photography. All photographs were taken at a magnification of $10 \times$.

We measured the dimensions of the facet joint area using ImageJ 1.52 (NIH, Bethesda, MD, USA). The facet joint area was defined as the cross-sectional area including the superior articular process and joint space on the selected slides. We compared the left and right facet joint areas by group.

\section{Footprint Test}

A footprint test was performed a day before surgery and a day before 4 weeks after surgery before the rats were euthanized, using the method described by Klapdor et al. ${ }^{29} \mathrm{~A}$ white paper was laid on the bottom of a prefabricated wooden box, which was designed as a small inclining gangway (Figure 3). The rats, with blue ink applied on the forefeet and red ink on the hindfoot, walked up the gangway into a dark compartment. All rats underwent three pre-training runs before the test to adapt to the box. We obtained footprints on the paper in the gangway, and the stride length of the hindfoot (red color) of each side was measured (Figure 4).

\section{Statistical Analyses}

All results are expressed as the mean \pm standard deviation. The differences in the left and right stride lengths and differences in the left and right facet joint areas were analyzed using the paired $t$-test.

Statistical significance was set at p-values $<0.05$. Statistical analysis was performed using R 4.1.1 (The University of Auckland, Auckland, New Zealand). 
A

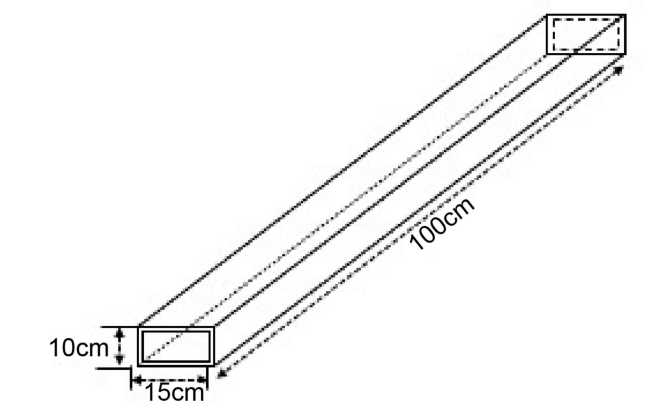

B

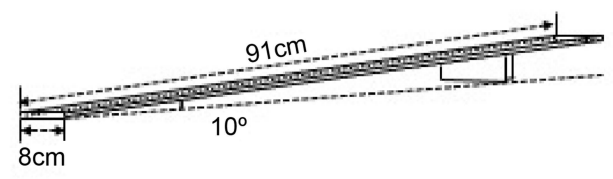

C

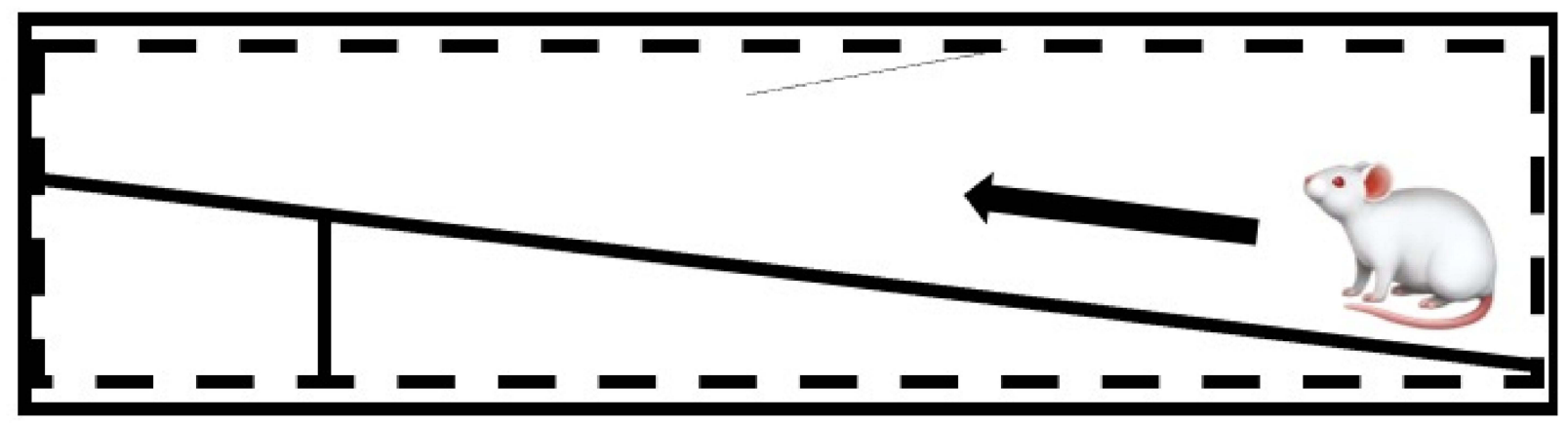

Figure 3 Schematic illustration of the prefabricated crate for the footprint test. (A) Schematic illustration of the crate for the rats to go through. (B) The ramp made for a rat to climb inside a crate. (C) The ramp was set in the crate. The rat leaves footprints as it climbs the ramp set in the crate.

\section{Results}

\section{Facet Joint Area}

The facet joint areas were measured using slide images stained with Masson's trichrome stain. The difference between the left and right facet joint areas was not statistically significant in group $\mathrm{A}(\mathrm{p}=0.50)$; however, the right facet joint area was significantly smaller than the left in group B $(\mathrm{p}<0.01)$ (Figure 5 and Table 1$)$. The differences between the left and right facet joint areas were $3413.4 \pm 11,392.24 \mu \mathrm{m}^{2}$ and $37,031 \pm 23,972.55 \mu \mathrm{m}^{2}$ for groups $\mathrm{A}$ and $\mathrm{B}$, respectively.

\section{Stride Length (from Footprint Test)}

None of the rats showed motor paresis or stress reactions during the experiment. There was no significant difference in the stride length 4 weeks after surgery. Group A had a greater stride length on the left than on the right before the surgery, but there was no significant difference 4 weeks after the surgery $(p=0.22)$. Group B did not show a significant difference between the right and left stride lengths both before and 4 weeks after surgery $(\mathrm{p}=0.46)$ (Table 2).

\section{Discussion}

In this study, unilateral facetectomy was performed within the right L5-6 area in rats. Using this experimental model, we were able to assess the degree of defect in the right ipsilateral facet joint area and compare it with the contralateral side. The comparison of the facet joint area 4 weeks after the surgery showed no significant difference between the left and right sides in group A, but the area on the right side was significantly smaller than that on the left side in group B. We interpreted these results as follows: the bony defect from the facetectomy had healed in group A, but not in group B, in which the bone wax was applied at the facetectomy site.

Bone fractures undergo a natural healing process through inflammation after they are induced. ${ }^{30,31}$ Schnideler et al suggested a four-stage model for the healing process of bone fractures: inflammation, soft callus formation, hard callus formation, and bone remodeling. ${ }^{32} \mathrm{~A}$ fractured bone is associated with a disruption of tissue integrity, an interruption of normal vessels, and a distortion of the marrow, which trigger the inflammatory processes in the wound healing pathway. Adequate blood flow to the bone is important for all phases of healing of a fractured bone. ${ }^{33}$ Bone healing in rats occurs over approximately 4 weeks, which is the period we set for observation in this study. ${ }^{34-36}$ 


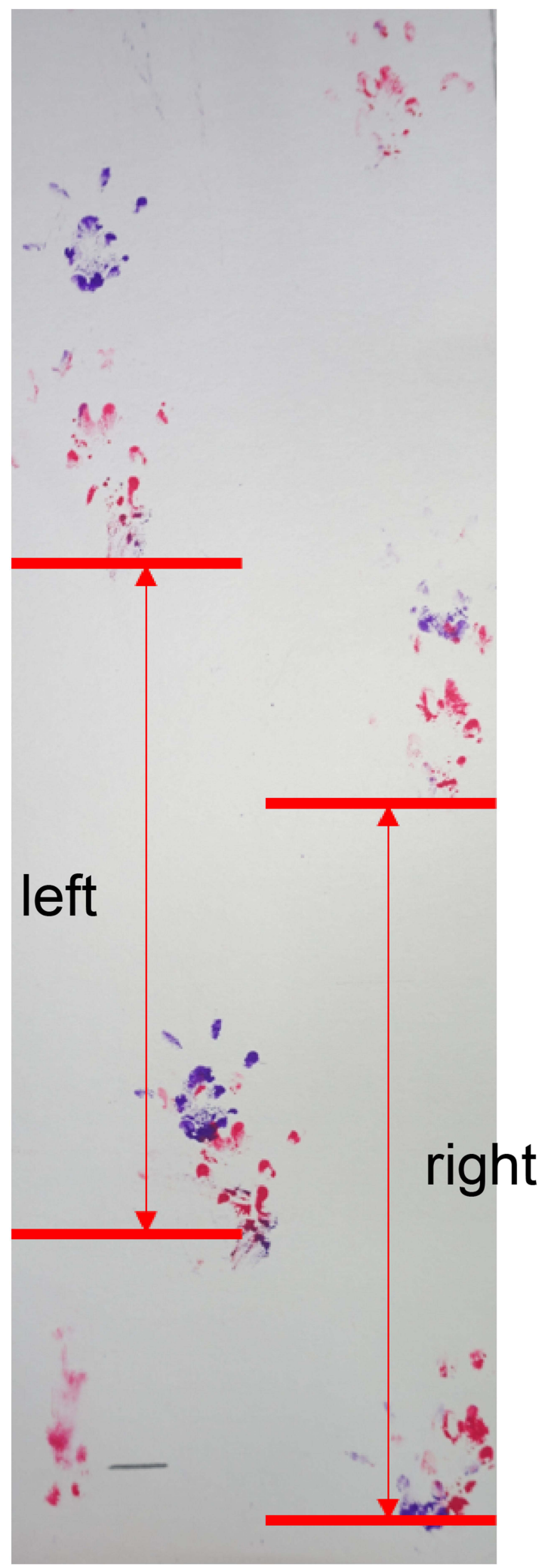

Figure 4 Measurement of stride length using footprint test. The blue footprints are for the forefoot, and the red footprints are for the hindfoot. Stride length is defined as the distance between the bases of the footprints of the hindfoot (red). The image is that of Sample \#4 from Group A. 

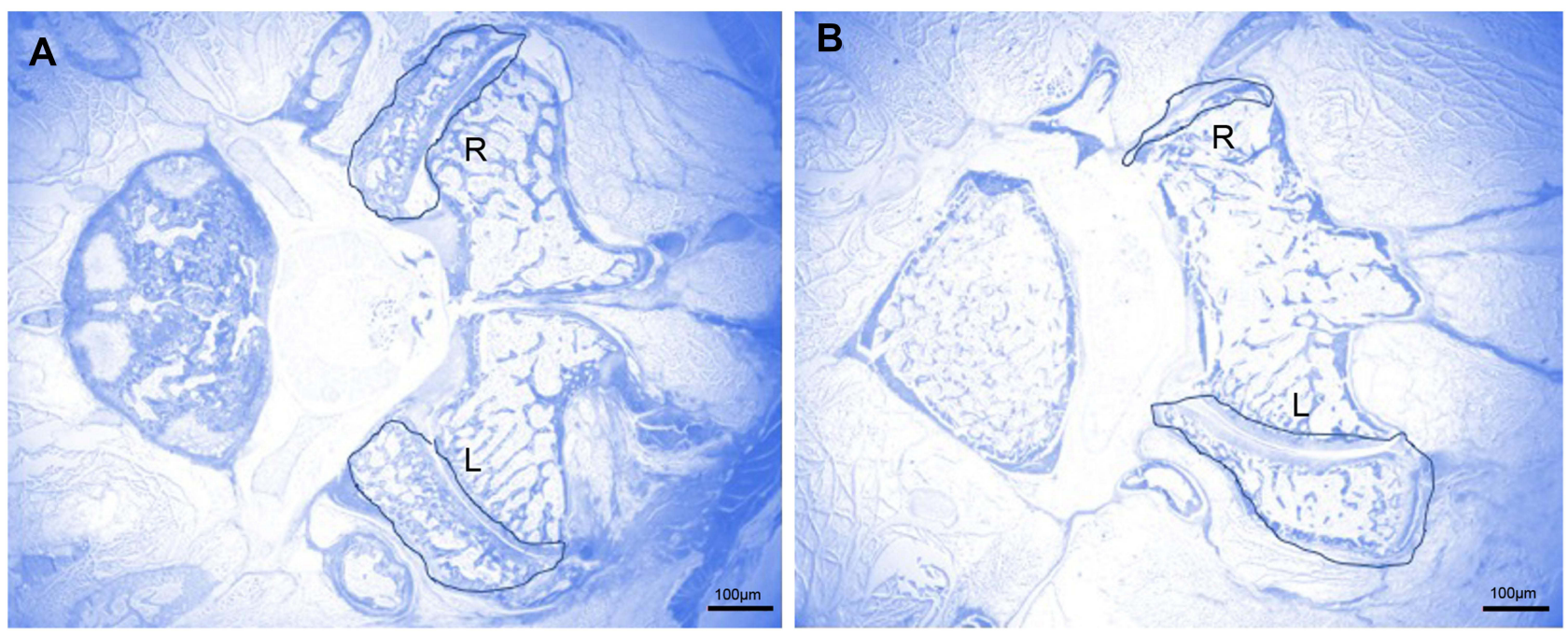

Figure 5 Microscopic (Eclipse TE300 inverted microscope, Nicon ${ }^{\circledR}$, Japan) images of slides at L5-6. The slides are made from the harvested lumbar spine at L5-6 4 weeks after surgery. The facet joint area includes the superior articular process and joint space (area in dark blue line). All photographs were taken using I0x magnification. The areas were measured using Imagej software. (A) Group A (control) and (B) group B (bone wax). The images are those of Sample \#6 from Group A and Sample \#I from Group B.

Abbreviations: L, left; R, right.

Bone wax controls bleeding from the bone by acting as a tamponade to stop blood flow from the damaged vessels in the trabecular bone, thereby facilitating clot formation. ${ }^{25}$ These characteristics can inhibit bone tissue regeneration after facetectomy and maintain the generated bony defect. We chose water-soluble bone wax, which did not leave any traces; this provides a similar environment to real bony defect sites. We observed that bone wax impeded the normal healing process of the bone and maintained the created defect without leaving any traces.

Bony defects at the facet joint are not uniform in shape; hence, it is difficult to evaluate the defect size. In this study, the defects were evaluated by comparing the sizes of the remaining facet joints by histological evaluation. This method is simple, convenient, and inexpensive. All laboratories that possess microscopes can perform this experiment, and we expect that experiments on bony defects in the facet joint can be performed with a lower barrier based on this model.

In this study, the left (uninjured) facet joint was larger in group B than in group A. The mechanism of this result is unclear. However, the authors hypothesize that it is a result of excessive bone remodeling by the bone cells, particularly

Table I Facet Joint Area (Unit: $\left.\mu \mathrm{m}^{2}\right)$

\begin{tabular}{|l|c|c|c|}
\hline & Left & Right & p-value \\
\hline Group A (Control) & $37,746 \pm 20,448.53$ & $34,333 \pm 23,954.25$ & 0.50 \\
Group B (Bone wax)* & $57,639 \pm 1 \mathrm{I}, 648.1$ & $20,609 \pm 23,676.48$ & $<0.01$ \\
\hline
\end{tabular}

Notes: The facet joint areas of selected slides (Figure 5) were calculated using Imagej. Numbers are shown as mean \pm standard deviation (SD). *Statistically significant difference between the left and right $(p<0.05)$.

Table 2 Stride Lengths from the Footprint Analysis (Unit: cm)

\begin{tabular}{|l|l|l|l|l|l|l|}
\hline & \multicolumn{3}{|c|}{ Before } & \multicolumn{3}{c|}{ 4 Weeks } \\
\cline { 2 - 7 } & Left & Right & p value & Left & Right & p value \\
\hline Group A (Control) & $10.27 \pm 0.93$ & $9.93 \pm 1.09$ & 0.02 & $10.27 \pm 1.27$ & $11.02 \pm 1.68$ & 0.22 \\
Group B (Bone wax) & $10.57 \pm 1.53$ & $10.53 \pm 0.92$ & 0.93 & $11.92 \pm 3.66$ & $11.10 \pm 1.6$ & 0.46 \\
\hline
\end{tabular}

Notes: The stride lengths were measured during the footprint analysis (Figure 4). Numbers are shown as mean \pm standard deviation (SD). 
osteoclasts and osteoblasts, which primarily heal the injured bony structure in normal conditions but cannot go to the injured site maintained with bone wax. ${ }^{24}$ Still, no study has investigated the effect on the surrounding bone tissue when bone healing is disturbed by bone wax, and this finding should be verified in future studies.

In a gait analysis, Fukui et al demonstrated that spinal instability induced by facetectomy resulted in abnormal walking patterns in rats, which may be caused by low back pain. ${ }^{19}$ The present study differs from the one by Fukui et al in two ways. First, unilateral facetectomy was performed to compare both sides; therefore, this may not have caused spinal instability that would affect gait, unlike previous experiments with bilateral facetectomy. The second difference was the observation time: Fukui et al compared the left and right stride lengths among the control (naïve rats), sham (only the bilateral facet joints at the L4-5 were exposed), and experimental (underwent complete resection of the bilateral L4-5 facet joints) groups at 3, 4.5, 6, and 7 weeks after facetectomy, and observed a difference at 3 and 7 weeks after surgery. If the rats were presumed to have developed acute to chronic pain 4 weeks after injury, the difference after 3 weeks may have been attributable to acute pain, and that at 7 weeks may have been attributable to chronic pain. ${ }^{37}$ We observed the results 4 weeks after the surgery, which was sufficient to see whether the bony defect was maintained. However, further research with a longer duration and involving a greater number of rats is needed for behavioral assessment using this model.

Our study had limitations related to the small sample size and short duration of observation. We found that the created defects in the facet joint were maintained at 4 weeks after surgery in group B, not in group A. However, the results from the footprint test did not show significant differences in either group in this study. This should be verified with further studies involving larger samples and a longer follow-up period. In addition, if available, more advanced tools (eg, CatWalk system) for gait analysis are valuable for validation of the model. ${ }^{21,38}$ Small sample size can cause a relatively large standard error in the results, such as the facet joint area in our study. Despite this limitation, differences in the facet joint area measurements between both sides had a normal distribution according to the normality test ( $\mathrm{p}$-value $>0.05$ in Shapiro-Wilk test); a paired $t$-test was available in both group A and B. The authors attempted to use X-ray or ultrasound to evaluate the defect sizes initially, but the imaging quality was not very good. MicroCT, which is likely the best for this kind of study, was not available to the authors. Despite this, we observed that the created bony defect was preserved by bone wax, which mimicked spondylolysis, using only a microscope. Our methods can thus reduce the barriers to conducting related studies and may help popularize them.

\section{Conclusion}

We presented a method for the induction and evaluation of bony defects following facetectomy at the facet joints of L5-6 using a rat model. We expect that the findings obtained in this study may be validated using microCT, larger samples, and longer follow-ups. Furthermore, we expect that this model may provide a basis for developing various treatments for bony defects at the level of the facet joints in the spine.

\section{Data Sharing Statement}

The data that support the findings of this study are available from the corresponding author on reasonable request.

\section{Ethics Approval}

The protocol for this study conformed to the guidelines of our institution for the care and use of laboratory animals. All study procedures were approved by the Institutional Review Board of Daejeon St. Mary's Hospital, The Catholic University of Korea (CMCDJ-AP-2016-007).

\section{Acknowledgments}

The authors thank the Catholic University of Korea Daejeon St. Mary's Hospital and The Catholic University of Korea Daejeon St. Mary's Hospital for their financial and non-financial support in conducting this study.

\section{Author Contributions}

All authors made a significant contribution to the work reported, whether that is in the conception, study design, execution, acquisition of data, analysis and interpretation, or in all these areas; took part in drafting, revising or critically 
reviewing the article; gave final approval of the version to be published; have agreed on the journal to which the article has been submitted; and agree to be accountable for all aspects of the work.

\section{Funding}

This work was supported by the Catholic University of Korea Daejeon St. Mary's Hospital and the Clinical Research Institute Grant funded by The Catholic University of Korea Daejeon St. Mary's Hospital (CMCDJ-AP-2016-007).

\section{Disclosure}

The authors declare that they have no conflicts of interest.

\section{References}

1. Manchikanti L, Boswell MV, Singh V, Pampati V, Damron KS, Beyer CD. Prevalence of facet joint pain in chronic spinal pain of cervical, thoracic, and lumbar regions. BMC Musculoskelet Disord. 2004;5(1):15. doi:10.1186/1471-2474-5-15

2. Lv B, Yuan J, Ding H, et al. Relationship between endplate defects, Modic change, disc degeneration, and facet joint degeneration in patients with low back pain. Biomed Res Int. 2019;2019:9369853. doi:10.1155/2019/9369853

3. Chung CC, Shimer AL. Lumbosacral spondylolysis and spondylolisthesis. Clin Sports Med. 2021;40(3):471-490. doi:10.1016/j.csm.2021.03.004

4. Micheli LJ, Wood R. Back pain in young athletes. Significant differences from adults in causes and patterns. Arch Pediatr Adolesc Med. 1995;149 (1):15-18. doi:10.1001/archpedi.1995.02170130017004

5. Cavalier R, Herman MJ, Cheung EV, Pizzutillo PD. Spondylolysis and spondylolisthesis in children and adolescents: i. Diagnosis, natural history, and nonsurgical management. $J$ Am Acad Orthop Surg. 2006;14(7):417-424. doi:10.5435/00124635-200607000-00004

6. Gagnet P, Kern K, Andrews K, Elgafy H, Ebraheim N. Spondylolysis and spondylolisthesis: a review of the literature. J Orthop. 2018;15 (2):404-407. doi:10.1016/j.jor.2018.03.008

7. Hirano A, Takebayashi T, Yoshimoto M, Ida K, Yamashita T. Characteristics of clinical and imaging findings in adolescent lumbar spondylolysis associated with sports activities. J Spine. 2012;1(124):2. doi:10.4172/2165-7939.1000124

8. Metzger R, Chaney S. Spondylolysis and spondylolisthesis: what the primary care provider should know. J Am Assoc Nurse Pract. 2014;26 (1):5-12. doi:10.1002/2327-6924.12083

9. Fujii K, Katoh S, Sairyo K, Ikata T, Yasui N. Union of defects in the pars interarticularis of the lumbar spine in children and adolescents. The radiological outcome after conservative treatment. J Bone Joint Surg Br. 2004;86(2):225-231. doi:10.1302/0301-620X.86B2.14339

10. Shamrock AG, Donnally IC, Varacallo M. Lumbar spondylolysis and spondylolisthesis. In: StatPearls. Treasure Island (FL): StatPearls Publishing; 2021.

11. Kalichman L, Kim DH, Li L, Guermazi A, Berkin V, Hunter DJ. Spondylolysis and spondylolisthesis: prevalence and association with low back pain in the adult community-based population. Spine. 2009;34(2):199-205. doi:10.1097/BRS.0b013e31818edcfd

12. Omidi-Kashani F, Ebrahimzadeh MH, Salari S. Lumbar spondylolysis and spondylolytic spondylolisthesis: who should be have surgery? An algorithmic approach. Asian Spine J. 2014;8(6):856-863. doi:10.4184/asj.2014.8.6.856

13. Moriguchi Y, Alimi M, Khair T, et al. Biological treatment approaches for degenerative disk disease: a literature review of in vivo animal and clinical data. Global Spine J. 2016;6(5):497-518. doi:10.1055/s-0036-1571955

14. Joshi HP, Jo HJ, Kim YH, An SB, Park CK, Han I. Stem cell therapy for modulating neuroinflammation in neuropathic pain. Int J Mol Sci. 2021;22 (9):4853. doi:10.3390/ijms22094853

15. Beadon K, Johnston JD, Siggers K, Itshayek E, Cripton PA. A repeatable ex vivo model of spondylolysis and spondylolisthesis. Spine. 2008;33 (22):2387-2393. doi:10.1097/BRS.0b013e318184e 775

16. Colloca CJ, Gunzburg R, Freeman BJ, Szpalski M, Afifi M, Moore RJ. Biomechanical quantification of pathologic manipulable spinal lesions: an in vivo ovine model of spondylolysis and intervertebral disc degeneration. J Manipulative Physiol Ther. 2012;35(5):354-366. doi:10.1016/j. jmpt.2012.04.018

17. Roberto R, Dezfuli B, Deuel C, Curtiss S, Hazelwood S. A biomechanical comparison of three spondylolysis repair techniques in a calf spine model. Orthop Traumatol Surg Res. 2013;99(1):66-71. doi:10.1016/j.otsr.2012.10.011

18. Sakamaki T, Sairyo K, Katoh S, et al. The pathogenesis of slippage and deformity in the pediatric lumbar spine: a radiographic and histologic study using a new rat in vivo model. Spine. 2003;28(7):645-650;discussion 650-651. doi:10.1097/01.BRS.0000051915.35828.17

19. Fukui D, Kawakami M, Yoshida M, Nakao S, Matsuoka T, Yamada H. Gait abnormality due to spinal instability after lumbar facetectomy in the rat. Eur Spine J. 2015;24(9):2085-2094. doi:10.1007/s00586-014-3537-y

20. Fukui D, Kawakami M, Cheng K, et al. Three-dimensional micro-computed tomography analysis for spinal instability after lumbar facetectomy in the rat. Eur Spine J. 2017;26(8):2014-2020. doi:10.1007/s00586-016-4920-7

21. Fukui D, Kawakami M, Matsumoto T, Naiki M. Stress enhances gait disturbance induced by lumbar disc degeneration in rat. Eur Spine J. 2018;27 (1):205-213. doi:10.1007/s00586-017-5243-z

22. D’Mello S, Atluri K, Geary SM, Hong L, Elangovan S, Salem AK. Bone regeneration using gene-activated matrices. AAPS J. 2017;19(1):43-53. doi:10.1208/s12248-016-9982-2

23. Schemitsch EH. Size matters: defining critical in bone defect size! J Orthop Trauma. 2017;31(Suppl 5):S20-S22. doi:10.1097/ BOT.0000000000000978

24. Sims NA, Gooi JH. Bone remodeling: multiple cellular interactions required for coupling of bone formation and resorption. Semin Cell Dev Biol. 2008;19(5):444-451. doi:10.1016/j.semcdb.2008.07.016

25. Das JM. Bone wax in neurosurgery: a review. World Neurosurg. 2018;116:72-76. doi:10.1016/j.wneu.2018.04.222 
26. Nooh N, Abdullah WA, Grawish Mel A, Ramalingam S, Javed F, Al-Hezaimi K. The effects of surgical and bone wax hemostatic agents on bone healing: an experimental study. Indian J Orthop. 2014;48(3):319-325. doi:10.4103/0019-5413.129451

27. Boivin GP, Hickman DL, Creamer-Hente MA, Pritchett-Corning KR, Bratcher NA. Review of $\mathrm{CO}_{2}$ as a euthanasia agent for laboratory rats and mice. J Am Assoc Lab Anim Sci. 2017;56(5):491-499.

28. Jeong JH, Jin ES, Kim JY, et al. The effect of biocomposite screws on bone regeneration in a rat osteoporosis model. World Neurosurg. 2017;106:964-972. doi:10.1016/j.wneu.2017.07.083

29. Klapdor K, Dulfer BG, Hammann A, Van der Staay FJ. A low-cost method to analyse footprint patterns. J Neurosci Methods. 1997;75(1):49-54. doi:10.1016/S0165-0270(97)00042-3

30. Kratzel C, Bergmann C, Duda G, Greiner S, Schmidmaier G, Wildemann B. Characterization of a rat osteotomy model with impaired healing. BMC Musculoskelet Disord. 2008;9(1):135. doi:10.1186/1471-2474-9-135

31. Simon AM, Manigrasso MB, O'Connor JP. Cyclo-oxygenase 2 function is essential for bone fracture healing. J Bone Miner Res. 2002;17 (6):963-976. doi:10.1359/jbmr.2002.17.6.963

32. Schindeler A, McDonald MM, Bokko P, Little DG. Bone remodeling during fracture repair: the cellular picture. Semin Cell Dev Biol. 2008;19 (5):459-466. doi:10.1016/j.semcdb.2008.07.004

33. Tomlinson RE, Silva MJ. Skeletal blood flow in bone repair and maintenance. Bone Res. 2013;1(4):311-322. doi:10.4248/BR201304002

34. Hankenson KD, Zimmerman G, Marcucio R. Biological perspectives of delayed fracture healing. Injury. 2014;45 Suppl 2(0 2):S8-S15. doi:10.1016/j.injury.2014.04.003

35. Bernhardsson M, Sandberg O, Aspenberg P. Experimental models for cancellous bone healing in the rat. Acta Orthop. 2015;86(6):745-750. doi: $10.3109 / 17453674.2015 .1075705$

36. Garcia P, Histing T, Holstein JH, et al. Rodent animal models of delayed bone healing and non-union formation: a comprehensive review. Eur Cell Mater. 2013;26:1-12; discussion 12-14. doi:10.22203/eCM.v026a01

37. Calvino B, Crepon-Bernard MO, Le Bars D. Parallel clinical and behavioural studies of adjuvant-induced arthritis in the rat: possible relationship with 'chronic pain'. Behav Brain Res. 1987;24(1):11-29. doi:10.1016/0166-4328(87)90032-5

38. Yi M-H, Liu YU, Umpierre AD, et al. Optogenetic activation of spinal microglia triggers chronic pain in mice. PLoS Biol. 2021;19(3):e3001154. doi:10.1371/journal.pbio.3001154

Journal of Pain Research

Dovepress

\section{Publish your work in this journal}

The Journal of Pain Research is an international, peer reviewed, open access, online journal that welcomes laboratory and clinical findings in the fields of pain research and the prevention and management of pain. Original research, reviews, symposium reports, hypothesis formation and commentaries are all considered for publication. The manuscript management system is completely online and includes a very quick and fair peer-review system, which is all easy to use. Visit http://www.dovepress.com/testimonials.php to read real quotes from published authors.

Submit your manuscript here: https://www.dovepress.com/journal-of-pain-research-journal 